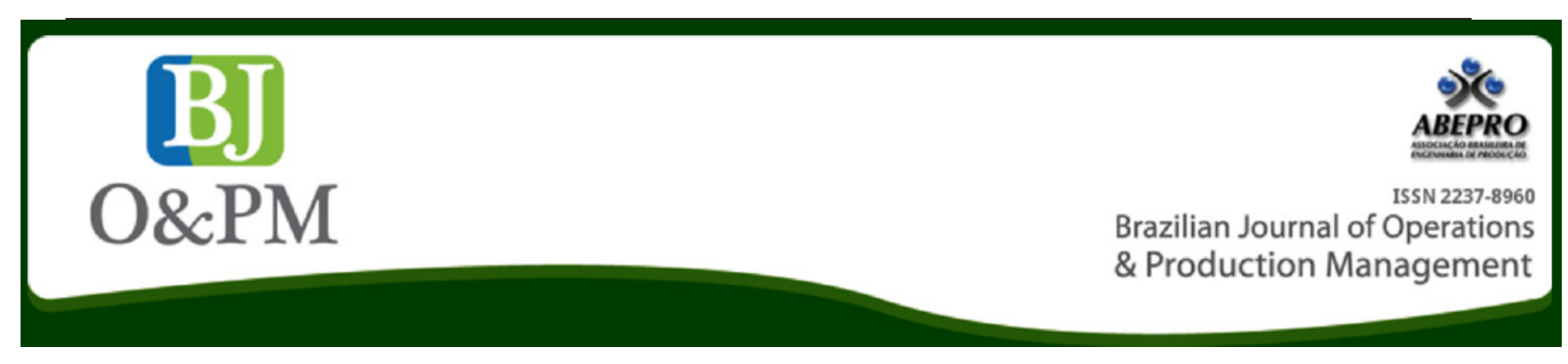

\title{
A TOOL TO EXPLAIN VISUALLY THE ZONES OF INFLUENCE OF SEVERAL DISTRIBUTION CENTERS IN A NETWORK
}

\section{Carlos Maligo}

\section{ABSTRACT}

Purpose - The concept of zone of influence is bound to arise in a great variety of situations involving explanations about supply chain, logistics or physical distribution of goods or services. Although the concept itself is not particularly difficult to explain or to understand, the classroom experience strongly recommends the use of drawing or any visual aid to obtain effective and fast results. This work is the result of the search for a tool which could not only provide a visual support that lecturers could easily use for their explanations but could also be capable of showing - visually - the effects on form and size of the zones of influence due to changes in the related variables.

Design/methodology/approach - The paper describes the construction of the tool - a set of spreadsheets - step by step. As the description progresses, the theoretical concepts involved are presented alongside with a practical example scenario. The scenario shows a typical automotive fuels distribution chain.

Findings - The truly visual output is the most rewarding result of this project regarding its original goal. Its simulation-like reactions and its capability to deal with low complexity real business scenarios are a plus.

Research limitations/implications - The tool deals with up to three zones of influence. No need to expand this capability is expected in the educational environment. Further analysis on the matter is needed concerning business applications, since exponentially growth of calculation complexity is to be expected.

Practical implications - The tool described in this paper provides a visual aid to explain the zone of influence concept. It can also be used as a cheap and simple to build simulation tool to help decision making processes in business environments.

Originality/value - The use of the conditional format command of the Microsoft Excel software made it possible to transform a spreadsheet in a simulation output display, capable of showing up to three zones of influence and its changings in accordance to variations in the model's related variables

Keywords: zone of influence, physical distribution, logistics, simulation, education. 


\section{INTRODUCTION}

The concept of zone of influence is well spread in our world and can be found in fields as diverse as ecology, medicine, agriculture, marketing, politics and logistics. The concept is rather simple and intuitive, and that is probably the reason of its usefulness to maybe any area of activity. Such a wide range of application implies the adaptation of the concept to each case, and according to them, the zone of influence focused can vary from being clearly physical, like the range of the daily wandering of a wild animal on its habitat, to virtual ones, like the possible shift in public opinion due to a certain action via social media.

In this work we are interested on the physical, geographic wise, concept, related to the power of businesses of reaching clients according to certain conditions and the influence that variations in these conditions may have on that reach. Small stores may focus on their neighbourhood; big malls know that easy access for car riding people is vital to attract the great numbers of clients they need; pizza deliveries depend on their delivery systems to survive a competition that can be next door. Knowing what determines the reach and how it behaves provides important information for a good logistics management.

The concept of zone of influence is not difficult to explain or to understand, particularly in its most simple example: one supplier and the clients it reaches, with some variables influencing that reach, like transportation cost. But imagine you have to explain the concept to an audience far from homogenous and sometimes with no background on logistics or on the industry you are focusing. Now imagine that besides that you will have to move past the concept and explain supply chains with several suppliers, several consumers and some steps in between. How would you ensure a good understanding of the concept and maintain their interest to show the relationships lying under the figures and how they influence each other? This work presents a solution, beginning by stating the objectives on section 2 and describing the method used to construct the proposed tool on section 3. Results obtained are addressed on section 4 and section 5 concludes this article.

\section{OBJECTIVES}

The objective of this work is to present a simple but efficient tool able to provide a visual aid to explain the concept of zone of influence. The tool was conceived and designed aiming logistics scenarios, but efforts were made to keep it as simple and generic as possible so it could be useful in a wide range of applications.

It should be kept in mind that the focus was on education. The simplicity of the tool was a key to ensure easiness of utilization with a minimum previous training of the instructor.
The use of well known standard software was important to ensure low cost and compatibility with the projection hardware commonly available at classrooms. The simplicity of the tool and the use of standard software should also provide easy adaptation to other fields, whenever necessary.

The tool was conceived and designed aiming a visual result capable of catching the attention of the public by being interactive, allowing the lecturer to show the effects on the zones of influence pictured due to changes in the related variables considered. This simulation-like characteristic is the heart of the idea, since it enhances the understanding of the concept as well as allows the explanation to go deeper into the complexities of real world examples.

\section{METHOD}

\subsection{Basics}

To achieve the objective a Microsoft Excel workbook was created. The workbook has several spreadsheets, each one adding one step to the construction of the final spreadsheet, which is the tool itself, and is the only one that needs to be displayed to the public. Having only one spreadsheet (the last one in the workbook) displayed right from the start, and considering that it doesn't show any tables, but a scenario which resembles a map with some points of reference and a few colored zones, enhances the simulation-like effects and the interactivity between the lecturer and the public via the tool. The construction of the workbook is described in the subsections ahead.

In Logistics, problem solving frequently involves determining the best alternative among several possibilities, evaluating the trade-off among their variables. Zones of influence can thus be explained as the area where a certain alternative beats all others according to some criteria, like minimum cost, maximum revenue, maximum profit, minimum distance, etc. In this work the minimum cost was the goal.

While there is strong evidence about the important role Logistics - and the related costs - play in the modern world, there is a broad range of options about how to assess and describe those costs. The breakdown used to keep track of the values should suit the firm's objectives or the analysis intended, but consensus is well established that total cost must be the main concern (Bowersox, 1996). In this work focus will be aimed on two very common and self-explaining cost components, since they are sufficient to demonstrate the zone of influence concept: transportation cost and operational cost of the installations.

Although only two cost components were used in this work, it should be emphasized that the tool is not limited to two variables and that the two components chosen are frequently listed among the most significant ones (Ballou, 
1992). This model was designed and executed according to one objective, and the example scenario that will be presented was its final goal, but it is expected that the conception and construction were kept sufficiently open so it could be applied to other cases or problems, by anyone interested.

Among typical logistics costs, transportation is usually listed as the biggest one (Bowersox, 1996). Accordingly, this work will highlight the comparisons between alternatives in terms of type of transport and different suppliers (different routes), the first represented by the freight rate and the latter by the corresponding distances. The other cost considered - operational cost - will be assigned to each installation. Thus, all distances, freight rate by type of transport and operational cost of all installations are input figures that the formulas embedded in every cell of the tool will use to produce the final result.

The tool handles the distribution of only one product. This was important to keep it as simple as possible since it allows using only one figure to each cost component. It should be mandatory to consider more than one product in the scenario, it is suggested to deal with one product per tool, and, if necessary, create another spreadsheet to summarize the partial results. Treating this last spreadsheet as the one and only tool will implicate bringing all input cells to it, which will require the modification of formulas and may result in visual pollution and difficulty of handling. It is suggested to ponder on the pros and cons, because perhaps creating another spreadsheet is not really necessary, and navigating among several spreadsheets is sufficient for the intended objective.

Using the idea and method proposed an example scenario was created illustrating a typical automotive fuels distribution chain (Author, 2005). This example comprises three terminals (T1 to T3), the main one receiving product from a refinery ( $R$ ) nearby, and six gas stations ( $R 1$ to $R 6$ ) randomly distributed on the scenario. The main terminal supplies fuel to the gas stations situated on its zone of influence and to the secondary terminal, which in turn supplies its zone of influence and the tertiary terminal. T3 supplies its zone of influence only. Transportation from terminals to gas stations is done by small trucks. Transportation between terminals can be done by rail or big trucks (Novaes, 2001). The construction of the workbook is described in the next subsections and is illustrated by images of the example scenario at the correspondent step of development.

\subsection{Background}

As previously stated, this work was primarily aimed to a physical distribution scenario, thus a geographic background is important. For this purpose, and since there is no need or interest in showing tables while using tool, the spreadsheets will be looked at essentially as maps, where the original grid available is going to work as a Manhattan metric grid. The Manhattan distance is the distance between two points measured along axes at right angles. (Black, 2006). In logistics, the Manhattan metric is often a simplification of the real problem which enables faster and simpler problem solving with reasonable precision. In this work, Manhattan metric is more than welcome, since it allows direct use of the spreadsheet's grid and is sufficient to explain the scenario with its map appearance, the reference points' locations and distances involved.

The first spreadsheet sets the background. Its default grid is going to represent a square grid of distances. A square grid is a particular case of a rectangular grid, but its use should not be interpreted as a limitation of the tool, since a rectangular grid can be easily set at this stage of the construction with just a few clicks. The choice of maintaining the default square grid was due to the fact that it was adequate for the purpose of this work and contributed to the tool's intended simplicity.

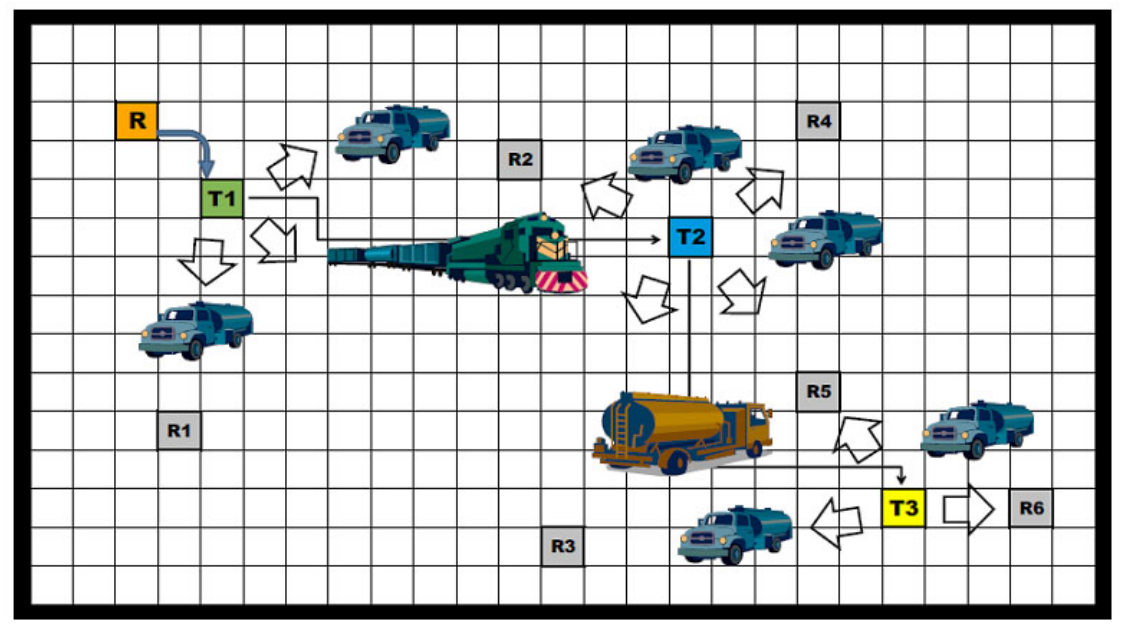

Figure 1 - The example scenario and the types of transportation available. Source: The authors own. 
Once the adequate grid is chosen - a square one, in this work - a cell's width (and height, since they are equal in a square grid) can be associated with a given distance, for example $20 \mathrm{~km}$. That distance is going to be the smallest subdivision on the map and, thus, should be adequate to set the desired scale to represent the scenario. Setting the scale is a virtual action; there is no associated formula to be inserted, command to be used or action to be taken on the spreadsheet. It is important to keep in mind the ratio "distance per cell" adopted to represent distances on the background, since locating objects on the scenario has to be coherent with this choice.

After choosing the adequate scale, the next step is to locate the reference points needed. These reference points can represent installations, infrastructure, borders, geographical accidents (rivers, coastline, mountains, etc.) and other "things" that could be useful to depict the scenario. It should be noted that for a theoretical explanation the tool can be prepared to show a simple and fictive scenario, but it is recommended that even in fictive scenarios care should be taken to set typical locations and distances as realistic as possible. This will help the public to associate the image they are seeing with their daily experience and enhance the simulation capacities of the tool when demonstrating the influence of the variables considered on the zones of influence portrayed.

Each reference point is going to be placed on a cell, so it can be named or tagged, and painted at will. It is recommended to represent all the reference points by a single cell whenever possible, since this simplifies the distance calculations. The cell may also be presented as the representation of the geographic center, the gravity center, the origin or the delivery point, etc., of bigger objects, in order to simplify the scenario. If in the case of using a group of cells representing an object is required,, it will be necessary to state and deal with some method to establish the adequate point from which to calculate the distances. This will be necessary only if the tool is going to be used as a simulator, and if a better precision is desired. Except in this case, the one cell method is strongly recommended.

The example scenario uses tags to identify the installations, as listed on Table 1 . These tags mark the location of the installations from the beginning of the construction of the tool, when the background is set, as showed on Figure 2.

Table 1. Reference points and correspondent tags on the example scenario

\begin{tabular}{ccc}
\hline Installation & Tag & Description \\
\hline Refinery & $\mathrm{R}$ & Refinery \\
Terminal 1 & T1 & Primary terminal \\
Terminal 2 & T2 & Secondary terminal \\
Terminal 3 & T3 & Tertiary terminal \\
Retail & R1 to R6 & Service stations \\
\hline
\end{tabular}

Source: Example scenario by the author.

\subsection{Distances}

Once the background is set, as described on the previous subsection, the next step is to calculate the distances. In the general case, it can be difficult to decide at this point which distances will be necessary to one's specific need. Thus, it is recommended to calculate every distance possible at once, which is not difficult to do, as it will be showed, and avoid having to return to this step every time a new distance is needed.

The default grid already available on the background provides direct measure of both horizontal and vertical distances. It suffices to multiply the number of cells between the desired two points by the scale previously set. Every other distance - neither horizontal nor vertical - is a hypotenuse of a right triangle that may be found on the grid. Since the two other sides - the catheti - of the right triangle are horizontal and vertical distances that can be measured as de-

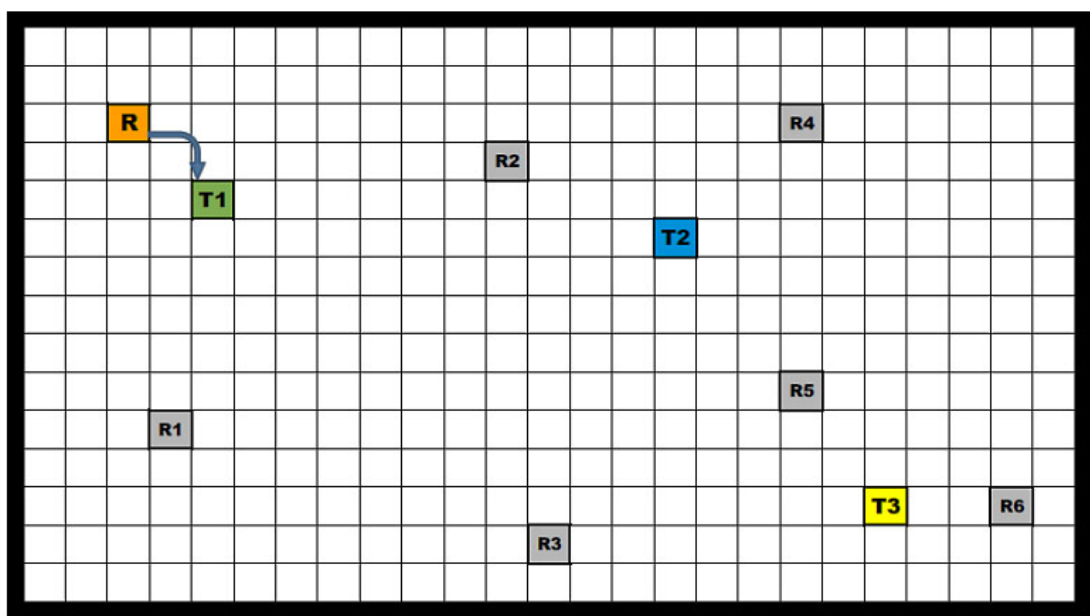

Figure $\mathbf{2}$ - The background spreadsheet for the example scenario. Source: The authors own. 
scribed above, the Pythagorean Theorem can be applied to find the length of the hypotenuse, i.e. the desired distance between the two points considered.

Knowing that to achieve the final result different options will be compared, it will be probably necessary to compute the distances from certain points to various other points. To do this, simply duplicate the background as many times as needed to create the sufficient number of spreadsheets to calculate a set of distances in each one, taking care to keep track of the alternatives created. Conveniently naming each spreadsheet may be helpful in the sequence.

In the example scenario four spreadsheets were necessary to register distances, three with the distances from each terminal and a fourth with the distances between Terminal 1 and Terminal 2 and between Terminal 2 and Terminal 3. The latter was necessary since the transference of product between terminals is what creates alternative routes and, thus, options to be chosen from. Figure 3 shows the distanc- es from Terminal 1 to the service stations. The distances in the horizontal and vertical axis passing through Terminal 1 are shown to act as a rule to facilitate visualization of the scale adopted $(20 \mathrm{~km} / \mathrm{cell})$ and the length of the catheti cited above. The distances to each cell are not shown in Figure 3 to avoid visual pollution of the figure and allow better understanding of this step of the tool's construction. Figure 4 shows all distances from Terminal 2.

\subsection{Transportation}

Transportation is a complex process and can be addressed to in various levels of detail according to the objectives and precision desired. In this work two significant variables related to transportation were considered: the type of transport employed and the distance travelled. The type of transport determines the freight rate, which multiplied by the distance of the route results in the cost to move one unit of the product through that particular route.

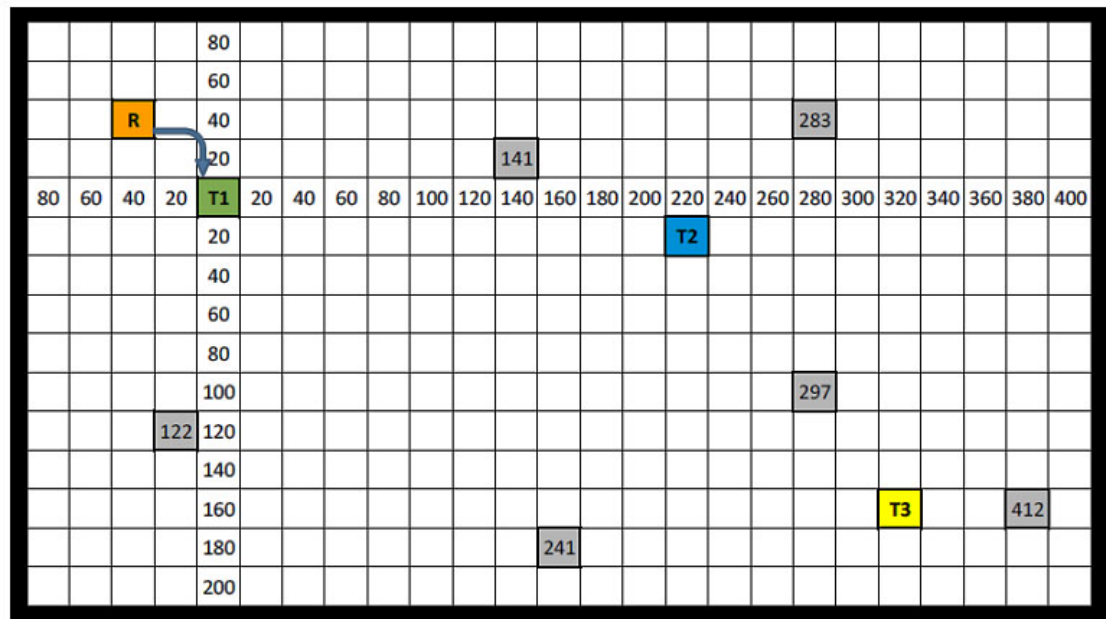

Figure 3 - Example scenario: distances from T1 to the service stations. Source: The authors own

\begin{tabular}{|l|l|l|l|l|l|l|l|l|l|l|l|l|l|l|l|l|l|l|l|l|l|l|l|l|}
316 & 297 & 279 & 260 & 242 & 224 & 206 & 189 & 172 & 156 & 141 & 128 & 117 & 108 & 102 & 100 & 102 & 108 & 117 & 128 & 141 & 156 & 172 & 189 & 206 \\
\hline
\end{tabular} \begin{tabular}{llllllllllll|l|l|l|l|l|l|l|l|l|l|l|l|l|l|l}
\hline 310 & 291 & 272 & 253 & 234 & 215 & 197 & 179 & 161 & 144 & 128 & 113 & 100 & 89 & 82 & 80 & 82 & 89 & 100 & 113 & 128 & 144 & 161 & 179 & 197 \\
\hline
\end{tabular}

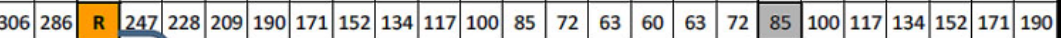
\begin{tabular}{llllllllllllllllllllllllll|l|l|l|l|}
303 & 283 & 263 & 243 & 24 & 204 & 184 & 165 & 146 & 126 & 108 & 89 & 72 & 57 & 45 & 40 & 45 & 57 & 72 & 89 & 108 & 126 & 146 & 165 & 184 \\
\hline
\end{tabular}

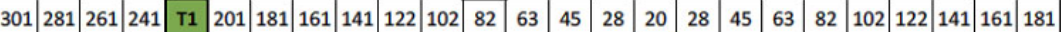
\begin{tabular}{|l|l|l|l|l|l|l|l|l|l|l|l|l|l|l|l|l|l|l|l|l|l|l|l|l|}
300 & 280 & 260 & 240 & 220 & 200 & 180 & 160 & 140 & 120 & 100 & 80 & 60 & 40 & 20 & T2 & 20 & 40 & 60 & 80 & 100 & 120 & 140 & 160 & 180 \\
\hline
\end{tabular}

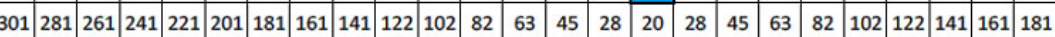
\begin{tabular}{lllllllllllllllllll|l|l|l|l|l|l|l|l|l|l|}
303 & 283 & 263 & 243 & 224 & 204 & 184 & 165 & 146 & 126 & 108 & 89 & 72 & 57 & 45 & 40 & 45 & 57 & 72 & 89 & 108 & 126 & 146 & 165 & 184 \\
\hline
\end{tabular}

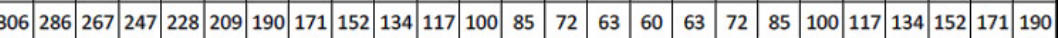
\begin{tabular}{ll|l|l|l|l|l|l|l|l|l|l|l|l|l|l|l|l|l|l|l|l|l|l|l}
310 & 291 & 272 & 253 & 234 & 215 & 197 & 179 & 161 & 144 & 128 & 113 & 100 & 89 & 82 & 80 & 82 & 89 & 100 & 113 & 128 & 144 & 161 & 179 & 197 \\
\hline
\end{tabular}

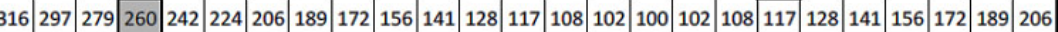
\begin{tabular}{llllllllllllll|l|l|l|l|l|l|l|l|l|l|l|l|l|l|}
323 & 305 & 286 & 268 & 251 & 233 & 216 & 200 & 184 & 170 & 156 & 144 & 134 & 126 & 122 & 120 & 122 & 126 & 134 & 144 & 156 & 170 & 184 & 200 & 216 \\
\hline
\end{tabular} \begin{tabular}{lllllllllllllllllllllll|l|l|l|l|l|l|l|l|}
331 & 313 & 295 & 278 & 261 & 244 & 228 & 213 & 198 & 184 & 172 & 161 & 152 & 146 & 141 & 140 & 141 & 146 & 152 & 161 & T3 & 184 & 198 & 213 & 228 \\
\hline
\end{tabular} \begin{tabular}{ll|l|l|l|l|l|l|l|l|l|l|l|l|l|l|l|l|l|l|l|l|l|l|l|l|}
340 & 322 & 305 & 288 & 272 & 256 & 241 & 226 & 213 & 200 & 189 & 179 & 171 & 165 & 161 & 160 & 161 & 165 & 171 & 179 & 189 & 200 & 213 & 226 & 241 \\
\hline
\end{tabular} \begin{tabular}{|l|l|l|l|l|l|l|l|l|l|l|l|l|l|l|l|l|l|l|l|l|l|l|l|l|}
350 & 333 & 316 & 300 & 284 & 269 & 255 & 241 & 228 & 216 & 206 & 197 & 190 & 184 & 181 & 180 & 181 & 184 & 190 & 197 & 206 & 216 & 228 & 241 & 255 \\
\hline
\end{tabular} 
Brazilian Journal of Operations \& Production Management

Volume 13, Número 3, 2016, pp. 276-284

DOI: 10.14488/BJOPM.2016.v13.n3.a5

The tool presented in this work has no limitation as for the modes of transportation or for the types of vehicles employed. Furthermore, creating a scenario with several modes of transportation and different types of vehicles do not increase the complexity of the tool as they will simply be represented by its corresponding freight rate, with no impact in the background or distances spreadsheets. The modes of transportation and the types of vehicles considered in any scenario are virtual items. They are not going to have any visual impact unless special elements related to them are introduced in one or more spreadsheets.

Nevertheless, it is important to keep track of the freight rates assumed at the start and afterwards, when demonstrating the impacts on the zones of influence caused by changes in the freight rates. To do so, some cells by the side of the scenario on the final spreadsheet (which is the tool itself, as previously explained) are sufficient. Each figure must occupy a different cell, as in a normal spreadsheet, since these cells are going to be referred to in the formulas which the tool will use in its calculations. Figure 5 shows how this was done in the example scenario.

\begin{tabular}{|c|c|c|c|c|c|}
\hline$\$$ & 10 & $/ \mathrm{m}^{3}$ & & & \\
\hline$\$$ & 12 & $/ \mathrm{m}^{3}$ & & & \\
\hline$\$$ & 15 & $/ \mathrm{m}^{3}$ & & & \\
\hline$\$$ & 0,05 & $/ \mathrm{m}^{3} \cdot \mathrm{km}$ & & \\
\hline$\$$ & 0,10 & $/ \mathrm{m}^{3} \cdot \mathrm{km}$ & \\
\hline$\$$ & 0,20 & $/ \mathrm{m}^{3} \cdot \mathrm{km}$ & \\
\hline
\end{tabular}

Figure $\mathbf{5}$ - Freight rate and operational costs input cells. Source: The authors own.

The freight rates are inputs to the tool. The scenario will show the zones of influence calculated with these figures. Since these values occupy cells that are referred to in the formulas that will determine each zone of influence, any change in any of these values will cause an immediate recalculation throughout the scenario, and the tool will present the new zones of influence at once.

\subsection{Operational cost}

The scenarios that may be used with the tool described in this work will probably include some installations, like, for example, warehouses, supermarkets, factories, retail stores, terminals and distribution centers. Determine the cost to operate each one may present difficulties ranging from the very definition as what to include under this label to its variation due to the type of installation, type of product, quantity of products handled, throughput, etc.

In this work the complexities related to assessing the operational cost were avoided. The tool only needs one input per installation, so it suffices, for example, to take an average monthly cost divided by the average monthly throughput. Using averages help keeping the simplicity of the tool, and considering the same period in time in the numerator and the denominator will result in values independent of time, expressing the cost to move one unit of product through each installation.

\subsection{Total cost}

The previous sections described how transportation and operational costs were calculated and expressed. Having both costs expressed in the same units - cost to move one unit of product through a route or installation - allows their direct addition to calculate the total cost. In a scenario with several routes and installations, there may be several ways of moving the product from origin to final destination, combining the different routes and installations available for the product to go through. Each combination represents an option and the tool will compare the options as for their total

\begin{tabular}{|c|c|c|c|c|c|c|c|c|c|c|c|c|c|c|c|c|c|c|c|c|c|c|c|c|}
\hline & & & & & 1 & & & & & & 2 & 36 & 39 & 43 & 47 & $s$ & 5 & 58 & 62 & 66 & 70 & 74 & 78 & \\
\hline & & & & 12 & 13 & 14 & & & & & 30 & 34 & 8 & 42 & 46 & 19 & 3 & 7 & 61 & 65 & 69 & 73 & 77 & \\
\hline & & & & $\varepsilon$ & & 11 & & & & & 29 & 33 & 37 & 41 & 12 & 49 & 53 & 57 & 61 & 64 & 68 & 72 & 76 & \\
\hline & & 9 & 6 & 4 & & & & & & & 8 & 2 & 6 & 10 & 44 & & & 6 & 60 & 64 & 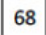 & 72 & 6 & \\
\hline & & 8 & 4 & T1 & 4 & & & & & & 28 & 32 & 36 & 40 & 44 & & 52 & 36 & 60 & 64 & 68 & 72 & 76 & \\
\hline & & 9 & 6 & 4 & 6 & 9 & 13 & 16 & 20 & 1 & 28 & 32 & 36 & 40 & & 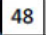 & 52 & 56 & 60 & 64 & 68 & 72 & 76 & 80 \\
\hline & & & 9 & 8 & 9 & 1 & & & & & 29 & 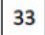 & 7 & 41 & 45 & & 53 & 7 & 61 & 64 & $\infty^{\circ}$ & 72 & 76 & \\
\hline & & & 13 & 12 & 13 & 14 & & & & & 30 & 34 & 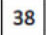 & 42 & 2 & 49 & 53 & 57 & 61 & 65 & 69 & 3 & 77 & \\
\hline & 0 & 18 & 16 & 16 & 16 & 18 & 20 & 23 & 26 & 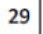 & 32 & 36 & 39 & 43 & 47 & 51 & 54 & 58 & 62 & 66 & 70 & 74 & 78 & \\
\hline & 2 & 2 & 20 & 20 & 20 & 22 & 23 & 26 & & & 34 & 38 & 41 & 45 & 48 & 52 & 56 & 59 & 63 & 67 & 71 & 75 & 79 & \\
\hline & & & & 24 & 24 & & & & & & & 40 & 43 & 47 & 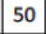 & 5 & 57 & 61 & 65 & 68 & 72 & 76 & 30 & \\
\hline 12 & 30 & 29 & 28 & 28 & 28 & 25 & 30 & 32 & 34 & 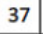 & 40 & 43 & 46 & 49 & 52 & 56 & 59 & 63 & 66 & 70 & 74 & 77 & 81 & 8 \\
\hline v & 34 & 33 & 32 & 32 & 32 & 33 & 34 & 36 & 38 & 0 & 43 & 45 & 48 & 51 & 54 & 58 & 61 & 64 & 68 & $D$ & 75 & 79 & 82 & \\
\hline & & & 3 & 3 & 36 & 37 & & & & & 46 & 48 & D. & 54 & | & 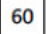 & 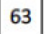 & 7 & 70 & 73 & 77 & 80 & 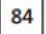 & \\
\hline & 42 & 41 & 40 & 40 & 40 & 41 & 42 & 43 & 45 & 47 & 49 & 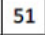 & D & - & 59 & - & 66 & 69 & ר? & 75 & 79 & & 86 & \\
\hline
\end{tabular}

Figure 6 - Example scenario: total costs delivering exclusively from T1.

Source: The authors own. 
cost. Figure 6 shows the simplest option: delivering exclusively from Terminal 1 to every cell.

The tool is ultimately a nodes and arcs model where each installation is a node and the routes are the arcs connecting them. This fact can be explored in adequate depth according to the logistics background of the audience.

\subsection{Colouring the scenario}

This work was interested in showing the zones of influence in a map-like fashion. That was possible by using the "conditional format" command of MS Excel and constructing an intermediate step between the calculations and the final result. This intermediate step compares the cost of each option, calculated in the previous spreadsheets, finds the cheapest, and assigns a code value to each cell. Each code value corresponds to one source installation, the origin of the route that yields the minimum cost. At the last spreadsheet the code value is used as input to the conditional format command to determine the color of the cell, thus painting all cells according to the best option to bring product to that point of the scenario. This is how the tool transforms the numerical results calculated in code values that, in its turn, will determine the color of the cell. Table 2 shows the relationships used in the example scenario.

Table 2. Relationship between installation, code value and colour

\begin{tabular}{ccc}
\hline Installation & Code value & Colour \\
\hline Terminal 1 & -1 & green \\
Terminal 2 & 0 & blue \\
Terminal 3 & +1 & yellow \\
\hline
\end{tabular}

Source: Example scenario by the author.

Up to three zones of influence can be showed simultaneously. The tool calculates the minimum cost option not only for the destination installations pictured, but also for every cell in the scenario. In that way, each cell sharing the same option will assume the same color, thus creating the desired effect: the scenario will show colored regions, each one being one zone of influence, as shown in Figure 7. This is why the figures used throughout the calculations, as well as the final results, are dispensable in the final spreadsheet, the tool itself.

This is the tool's specific contribution: provide an analysis of the entire scenario and show the zones of influence in the map. A few inputs and the previously prepared tool is all that is needed. The result is clearly visible and the influence of the variables may be easily demonstrated changing the inputs and observing how the boundaries of the colored regions move. This is the simulation-like effect intended and will be explored in the next section.

\subsection{Simulating}

As described at the end of subsection 3.1, the example scenario comprises three terminals and six gas stations. Transportation from terminals to gas stations is done exclusively by small trucks. Transportation between Terminals 1 and 2 is done by rail. Transportation between Terminal 2 and 3 is done by big trucks. There are three options to supply the gas stations: directly from $\mathrm{T} 1$, from $\mathrm{T} 1$ via $\mathrm{T} 2$ and from $\mathrm{T} 1$ via T2 and T3. The tool calculates the minimum cost option for each cell and colors it with the correspondent terminal's colour (Fig. 7).

From the initial state, any change in an input may produce changes in the zones of influence, moving the borders between them. Since MS Excel recalculates the entire scenario immediately after a new figure is introduced in any input cell, the effects of this change appear at once. The im-

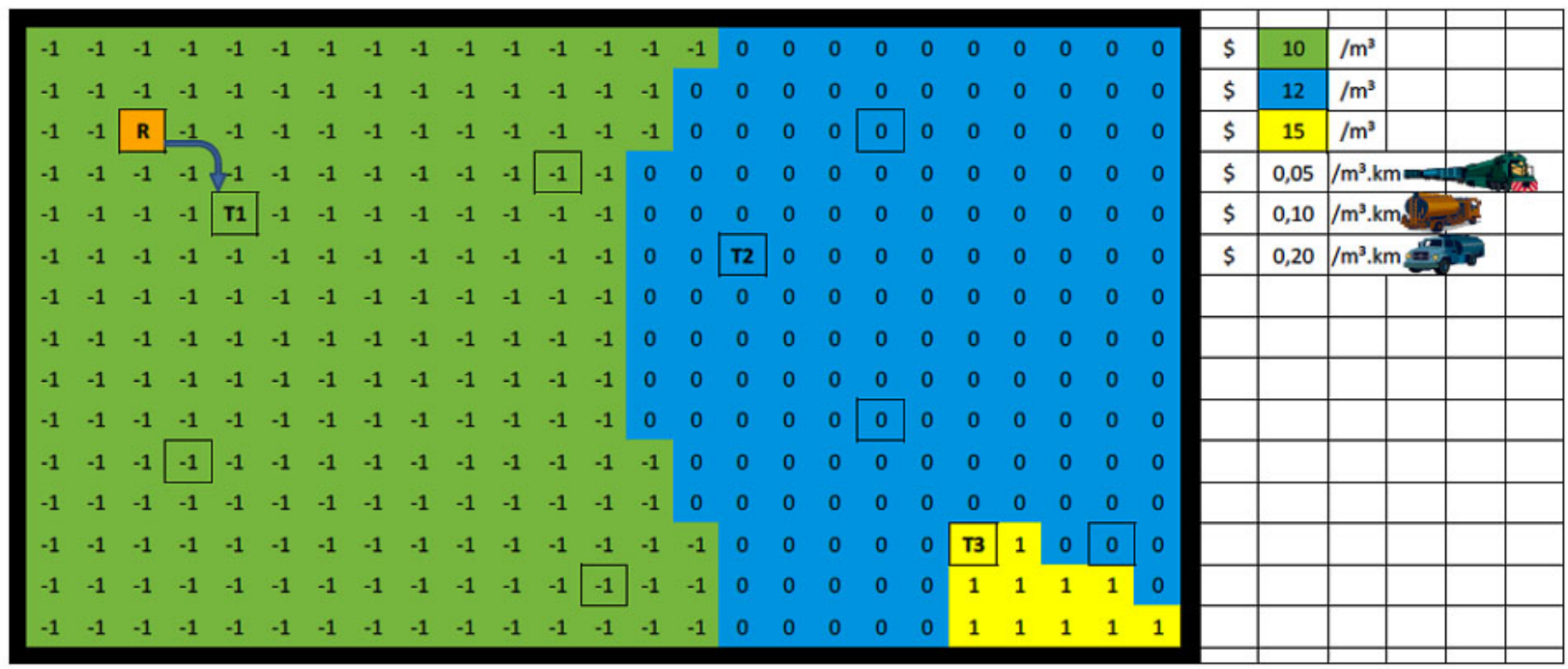

Figure 7 - The example scenario ready to use. Source: The authors own. 
Brazilian Journal of Operations \& Production Management

Volume 13, Número 3, 2016, pp. 276-284 DOI: 10.14488/BJOPM.2016.v13.n3.a5

mediate response of the scenario to any change in the input figures is an important asset of the tool. It provides the "real time" sensation to the audience and allows a big number of simulations to be done in just a few minutes. Clicking the "undo" and "redo" buttons on the software's main menu emphasizes the moving effect, highlights the cells which changed its colour and enlightens further analysis.

Determining the zones of influence for the entire scenario is another interesting asset of the tool. Calculate them for the gas station's cells inform the best route to supply the existing installations. Calculating them for the entire scenario means that it is possible to know the best route to supply any destination - a future installation, for example - with no additional effort. A prospect can therefore be located in the scenario and analysed to determine its supplying terminal and cost in advance. If the cells are associated to counties - and a real map is not needed for this, just real distances - the tool shows an unsuspected power to extrapolate its initial objective: more than a classroom aid, it has the capability to be used as a real simulation tool.

It is quite obvious, in the example scenario that the zone of influence of T3 would grow should the route passing through it become cheaper. How much cheaper it has to be to "capture" the R6 gas station (see Fig.1 for R6 location)? Which variables should go down and by what amount? These are questions that are much easier to answer with the aid of the proposed tool.

Exploring the tool's fast response, it is possible to find in seconds, in a simple trial and error way, that for an operational cost of $\$ 13.3 / \mathrm{m}^{3}$ at T3 the R6 gas station will be "captured" by this terminal's zone of influence. Since adding R6's volume to T3 helps lowering this terminal's initial operational cost $\left(\$ 15 / \mathrm{m}^{3}\right)$, it may be a good idea to recalculate T3's new operational cost with this additional volume, since the numbers seem to be converging.

On the other hand, finding the necessary drop in transportation cost from T2 to T3 to change R6 from T2's to T3's zone of influence is still easier: a $10 \%$ drop from the original $\$ 0.1 / \mathrm{m}^{3}$ to $\$ 0.09 / \mathrm{m}^{3}$ will do, as shown in Figure 8 . This specific finding shows how the simulation power of the tool can be useful in the real world. Knowledge of this relationship between transportation cost and zone of influence could lead to a successful win-win renegotiation of freight rate with the big trucks service provider: more cargoes at a cheaper unit cost, a typical logistics trade-off.

Whether it is advisable to change R6's supplying terminal from T2 to T3 will require deeper research. Forecasting better service level due to receiving from a nearer terminal in R6's manager point of view and calculating the rise of transportation cost by small trucks due to loss of mileage (maybe negligible) are examples of other aspects of the problem that should be addressed in a decision making process in the real world.

It is expected that the examples described in this subsection demonstrate the interesting features and capabilities of the proposed tool not only to achieve its objectives, but also to act as a simulator, simple but powerful, mainly with respect to preliminary analysis that could rapidly show the most important relationships to guide further calculations.

\section{RESULTS}

The tool described above was already used in two classes on logistics applied to the fuels distribution market in Brazil. Both classes were part of free courses aimed at people wishing to learn the basics of the oil industry. One of the classes

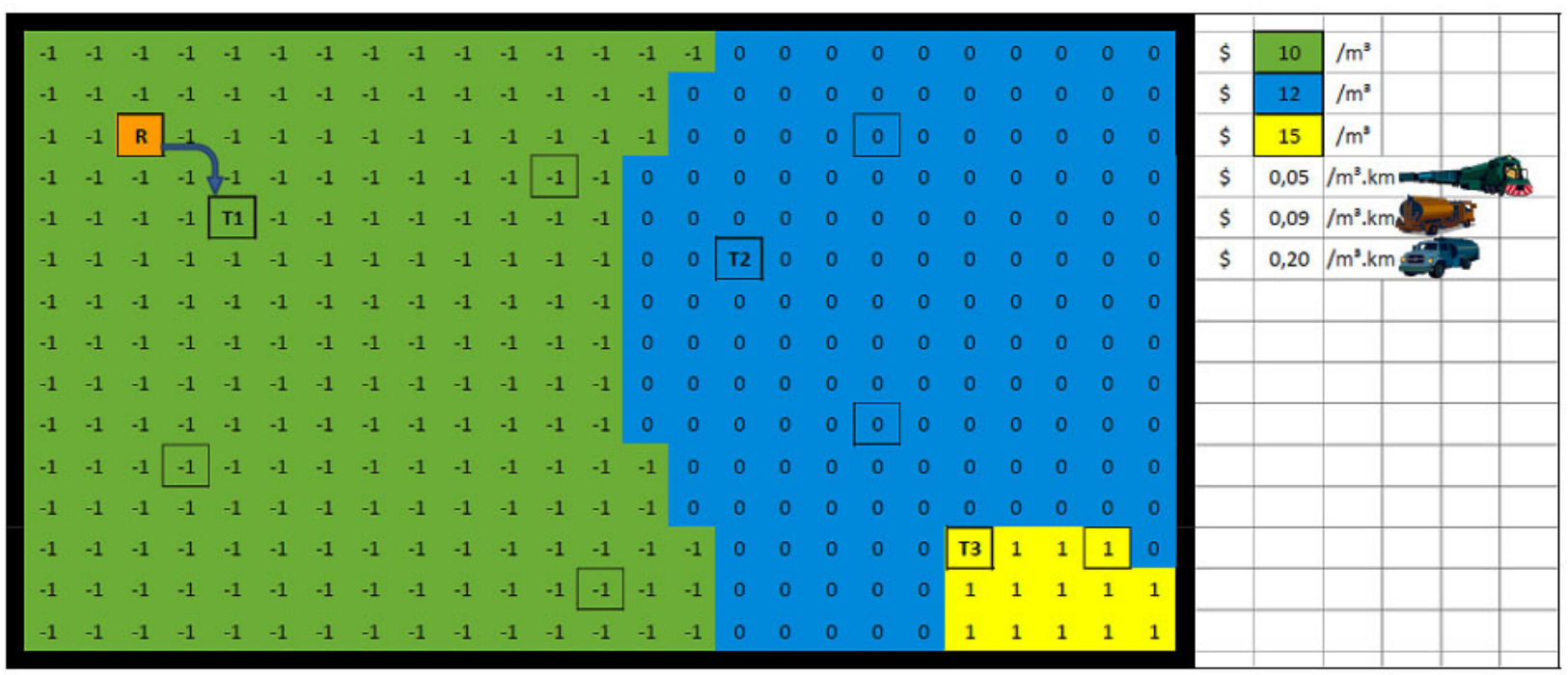

Figure 8 - The example scenario: effects of lowering big truck's transportation cost. Source: The authors own. 
was held at a very important university and the other at the most important Brazilian oil industry trade association, both in Rio de Janeiro. The public addressed was, thus, heterogeneous, mostly graduated professionals from a well spread range of careers, like engineering, administration, finance and law.

The results were most rewarding, since the tool provided just the visual way of teaching desired. The colourful images were as efficient in a classroom as in a much bigger auditorium. The interactivity with the tool easily captured the attention of the public, which realized at once that colours were not there just to garnish some presentation slides, but to show - in a real time way - the effects in the zones of influence caused by each change simulated in the costs variables. The interest of the public, evaluated by the number of smart questions, immediate associations with other scenarios and other markets, and eagerness to suggest other simulations or different changes in the variables to see what was going to happen, brought a strong confidence on the usefulness of the tool.

The construction and testing showed that the tool has potential to deal with harder problems. It is expected that utilisation of the tool in new opportunities and different environments could reveal new applications and demand for new developments.

\section{CONCLUSION}

The proposed tool is easy to construct, since it does not demand programming skills that a common computer user would not have, and requires only the hardware already available in today's classrooms, auditoriums and offices. It is inexpensive, consuming few working hours to be constructed and demanding only usually available hardware. The tool is easy to handle during a presentation and provides a nice visual aid to lecturers dealing with supply chain, logistics or physical distribution subjects. Furthermore, it brings better understanding of the relationship between the variables involved as well as allows deeper analysis through its simulation abilities.

\section{REFERENCES}

Ballou, Ronald H. (2006), Gerenciamento da cadeia de suprimentos / logística empresarial, 5 ed., Bookman, Porto Alegre, RS.

Bowersox, D.J., Closs, D.J. (1996), Logistical management: the integrated supply chain process, 1 ed., McGraw-Hill.

Black, Paul E. (2006), "Manhattan distance", in Dictionary of Algorithms and Data Structures, ed. 31/05/006, available at: http://www.nist.gov/dads/HTML/manhattanDistance. html (Accessed on 27/11/2015).
Author (2005)

Novaes, Antonio Galvão (2001), Logística e gerenciamento da cadeia de distribuição: estratégia, operação e avaliação, Campus. 